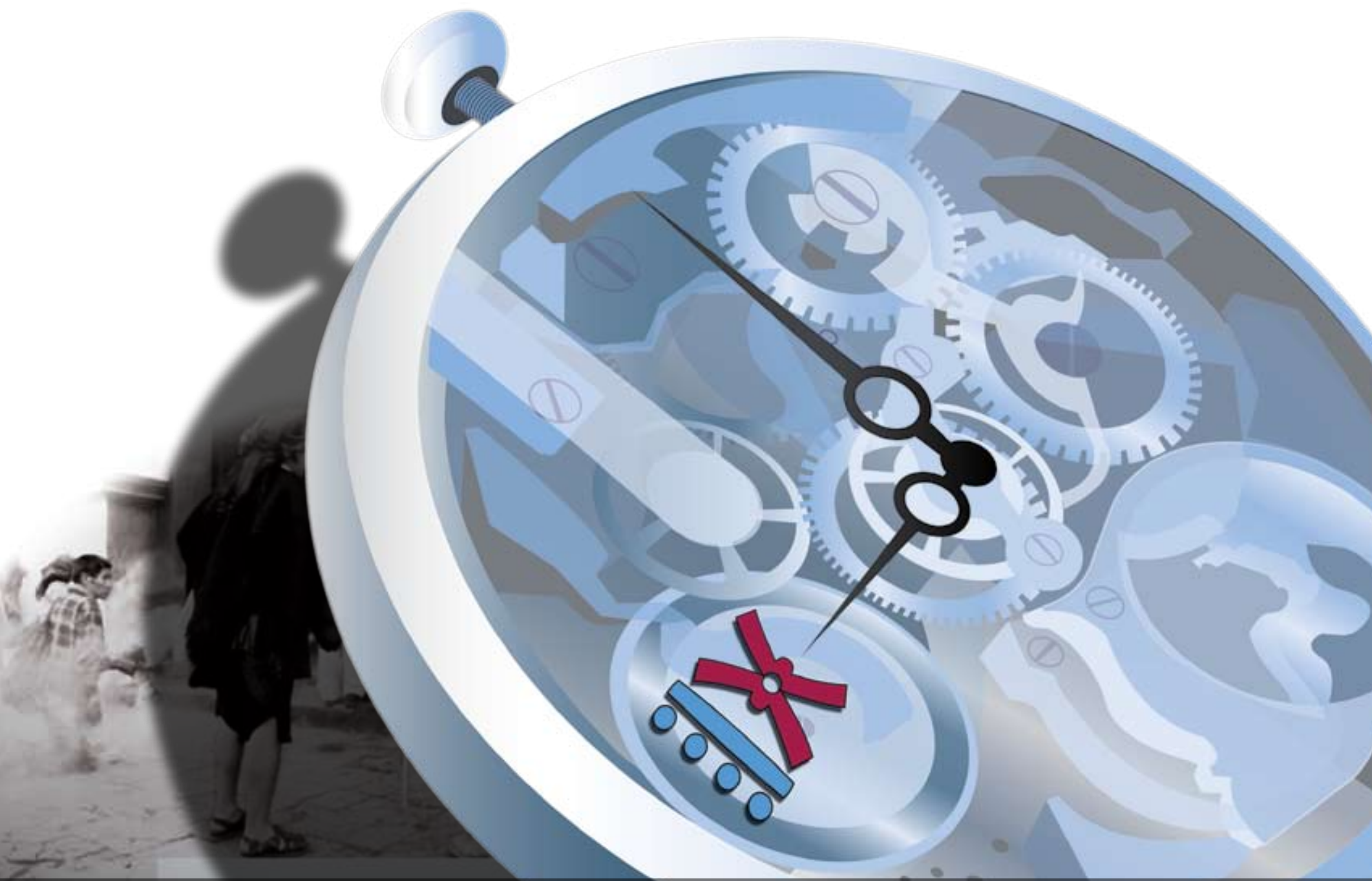

$9^{\circ}$ C O N G R E S O 2. CENTROAMERICANO DE H IS TO R I A
Universidad de Costa Rica

ISSN 1409-469X

Fecha de recepción: 15 de mayo 2008 Fecha de aceptación: 30 de mayo 2008

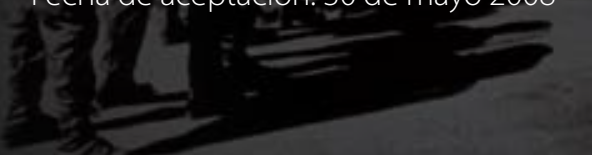

Elites y Médicas: Un análisis de las académicas en la Academia Nacional de Medicina de México

Miembros del Consejo Editorial:

Dr. Ronny Viales, Dr. Juan José Marín

Editores Técnicos:

Allan Fonseca, Andrés Cruz, Gabriela Soto

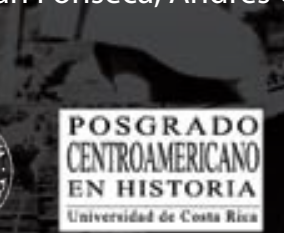




\section{Elites y Médicas: Un análisis de las académicas en la Academia Nacional de Medicina de México}

\section{Doctora Ana Cecilia Rodríguez de Romo}

Laboratorio de Historia de la Medicina, Instituto Nacional de Neurología y Neurocirugía, Manuel Velasco

Suárez

Departamento de Historia y Filosofía de la Medicina, Facultad de Medicina, UNAM ceciliar@servidor.unam.mx

\section{Maestra Gabriela Castañeda-López}

Laboratorio de Historia de la Medicina, Instituto Nacional de Neurología y Neurocirugía, Manuel Velasco

Suárez

gcasta95@yahoo.com.mx

Instituto Nacional de Neurología y Neurocirugía, Manuel Velasco Suárez. Laboratorio de Historia de la

Medicina. Avenida Insurgentes Sur 3877 Colonia la Fama, Delegación Tlalpan,

C. P. 14269, México, D. F.

Tel. 5606-3822 ext. 5032

Fax: 5528- 8036 


\section{Elites y Médicas: Un Análisis de las académicas en la Academia Nacional de Medicina de México $^{1}$}

\section{Introducción}

Tomando como modelo de estudio a las académicas, esta investigación analiza las características del grupo de mujeres que pertenecen a la Academia Nacional de Medicina de México (ANMM), una asociación médica de élite. Cabe señalar que nuestro campo de trabajo en las humanidades médicas no son los estudios de género, pero dos factores motivaron la realización de esta investigación. 1) La mayoría de los trabajos sobre mujeres que circunstancialmente caen en nuestras manos, principalmente abordan los problemas de los estratos marginados o desprotegidos de la sociedad, sin embargo, no son numerosas las investigaciones cuyos resultados se refieran a las mujeres en situación opuesta, es decir, aquellas que constituyen grupos especiales en la ciencia, la economía, la política o las humanidades. Evidentemente este grupo, por ejemplo, las médicas exitosas, no tiene los problemas que sufren campesinas, reclusas, iletradas, madres niñas o adolescentes, pero se ven confrontadas a otras conflictivas, producto de su incursión en una disciplina cuya identidad la historia ha definido como masculina. ${ }^{2}$ 2) La comunidad femenina de la ANMM, constituye un buen modelo para estudiar élites femeninas, pues es un grupo pequeño, compacto y uniforme, de reciente origen, considerando que la ANMM fue creada en 1864 y que la primera mujer ingresó hasta 1957.

El análisis fue enriquecedor, porque no hay estudios de élites médicas femeninas y porque puso en evidencia situaciones poco conocidas, muy significativas y semejantes a las que otros investigadores encontraron en las élites de ingenieras y de empresarias. ${ }^{3}$

En la actualidad las mujeres en las escuelas de medicina constituyen más de la mitad de la población total, sin embargo, no han incidido de igual manera en las posiciones de liderazgo o

$1 \quad$ Resultados parciales de este trabajo aparecerán publicados en la Gaceta Médica de México.

2 Teresa Ortiz-Gómez, "Fuentes orales e identidades profesionales: Las médicas españolas en la segunda mitad del siglo XX”, Asclepio 57, n. 1 (2005): 87.

3 Gina Zabludovsky, "Mujeres ejecutivas y empresarias en México", en Humanismo, mujer, familia y sociedad, comps. Mariano García-Viveros y Angélica Pulido (México, Sociedad Internacional Pro-valores Humanos E. Fromm-S. Zubirán, Programa Interdisciplinario de Estudios de la Mujer, El Colegio de México, Instituto Nacional de la Nutrición Salvador Zubirán, 1996), 206-234 y Patricia García Guevara, "El género y la carrera de las ingenieras ejecutivas: recortes de historias de vida y trayectorias", en Curso de vida y trayectorias de mujeres profesionistas (México, El Colegio de México, Programa Interdisciplinario de Estudios de la Mujer, 2007), 83-116. 
poder en la disciplina. Por una lado habrá que esperar a que esa joven población alcance la edad activa para insertarse en el medio, pero por otro, la razón no siempre está en la parte externa, parece que con frecuencia se debe a una decisión propia en el afán de congraciar las actividades profesionales con las personales.

\section{La Academia Nacional de Medicina de México, breve historia}

El primer antecedente en la historia de la ANMM se remonta a 1836, ${ }^{4}$ ese grupo inicial se disuelve pero quedan sus rastros en los seis volúmenes del Periódico de la Academia de Medicina de México. Dentro de los objetivos culturales de la intervención francesa, estuvo conformar en 1864 la Sección Médica de la Comisión Científica, Literaria y Artística, que perseguía estimular entre los mexicanos el interés por la ciencia y las bellas artes. En diciembre de 1865, esta Sección Médica se separó de la comisión y se constituyó en la Sociedad Médica de México. Aunque en los documentos y actas se autonombraba Academia, es hasta 1873 que se formaliza el nombre de Academia de Medicina de México. El año de 1877 es crucial porque entonces adquirió el carácter de nacional y el Congreso de la Unión le otorgó un subsidio anual incluido en la Ley de Presupuestos, lo que significó el reconocimiento de la ANMM por el gobierno.

A lo largo del siglo XIX, la ANMM tuvo un papel relevante en la introducción y difusión de la medicina científica en México. En sus sesiones, los médicos discutían lo último de su disciplina a la luz de su propia experiencia, y en su órgano de difusión, la Gaceta Médica de México, leían y publicaban también lo más novedoso.

En 1912, la ANMM fue reconocida oficialmente como Cuerpo Consultivo del Gobierno Federal. Sin embargo, desde 1910 y casi hasta 1925 las cosas no fueron fáciles, pues como consecuencia de la lucha civil producto de la Revolución, su lugar físico cambió varias veces aunque la mayoría del tiempo, se localizó en la Antigua Escuela de Medicina, conocida actualmente como Palacio de Santo Domingo en el centro histórico de la ciudad de México. A partir de 1956 la ANMM comprendió la necesidad de ampliar su radio de acción y creó las Jornadas Médicas y los Congresos que involucran a médicos de todo el país, poniendo así de manifiesto su verdadero carácter nacional. En 1961 el Instituto Mexicano del Seguro Social, IMSS, le asignó a la ANMM un enorme espacio en su Unidad de Congresos del Centro Médico Nacional para que fuera su sede. En 1985 y como consecuencia del sismo que afectó a la capital del país, la ANMM 4 Academia Nacional de Medicina [1864-1995]), 131 años al servicio de la salud (México, Academia Nacional de Medicina, 1995), 3-19. 
fue invitada a sesionar en el los auditorios del Hospital General y del Instituto Nacional de Cardiología, retornando a la Unidad de Congresos del IMSS, una vez que esta fue reparada.

En la actualidad, la ANMM cuenta con cuatro departamentos y 59 áreas de trabajo, y para el cumplimiento de sus actividades ha integrado quince Comités y Grupos de Estudio. De acuerdo a lo establecido desde 1864, sigue sesionando los miércoles a las ocho de la noche. Las sesiones son ordinarias, extraordinarias o solemnes y están abiertas al público, excepto cuando se declaran secretas. Deben ser presididas por el presidente o el vicepresidente si el primero faltara. La Gaceta Médica de México se ha publicado ininterrumpidamente desde 1864. La ANMM mantiene relación con diversas academias médicas en el mundo.

\section{Las primeras académicas}

Desde 1864, ser parte de la ANMM ha significado reconocimiento, prestigio y de cierta forma, pertenecer a la élite médica.

En 1887 se gradúo la primera mujer de médico cirujano por la Universidad Nacional de México, la doctora Matilde Montoya y Lafragua (1859-1938) [ver nota biográfica] sin embargo, es hasta 1957 que ingresó una mujer en la ANMM, la doctora Rosario Barroso Moguel (1923-2006) [ver nota biográfica]. La siguiente fue aceptada en 1965, la doctora Julieta Calderón de Laguna (1918-2001) [ver nota biográfica] y para 1973 se incorporó la tercera, la doctora María de la Soledad Córdova Caballero (1929-vive). Desde entonces y muy lentamente, se han abierto las puertas de la ANMM a las mujeres. A partir de 2000 su inserción puede considerarse numerosa. Entre los años 1887 y 1957, el único antecedente que da cuenta de la relación de las médicas con la ANMM, es una serie de cartas escritas en 1927, por el entonces presidente, doctor Everardo Landa y la doctora Antonia L. Ursúa, presidenta de la "Asociación de Médicas Mexicanas”. ${ }^{5}$ Landa le pidió a Ursúa los nombres y domicilios de las asociadas que eran quince, para invitarlas a asistir a la sesión inaugural del año académico. El doctor Landa se refiere a la doctora Ursúa como "muy estimable señorita", en lugar de "muy estimable doctora".

La ANMM tiene un reglamento para ingresar y un comité de admisión que discute los casos, pero la documentación que consigna las reuniones no es accesible para su consulta. Sería ilustrativo conocer que se dijo de la solicitud de Rosario Barroso, la primera académica, y si antes o después de ella hubo otras mujeres que pidieron entrar, recuérdese que transcurrieron once años desde 5 Expediente del doctor Everardo Landa. Archivo Histórico de la Academia Nacional de Medicina de México, ANMM. 
ella hasta la siguiente mujer aceptada.

En otros países se ha estudiado la admisión de las mujeres en las sociedades médicas y aunque el contexto es diferente, los casos muestran que el tema causaba conflictos. En Estados Unidos empezaron a graduarse doctoras en medicina a partir de 1849. Entre los problemas que provocó este hecho, había que decidir si al convertirse en médicas, las mujeres podían ser reconocidas como miembros del gremio y por lo tanto debían ser aceptadas en las sociedades. ${ }^{6}$ Seguir los debates es muy atractivo pues tanto los argumentos a favor como en contra, son perjudiciales para la parte femenina. En resumen, los partidarios de su ingreso apuntaban que eso fortalecería a la medicina ortodoxa, sobre la práctica “irregular” de los homeópatas o las comadronas. Los que estaban en contra se apoyaban en la supuesta menor capacidad de las mujeres y el pobre nivel académico de sus escuelas de medicina. La Sociedad Médica de Massachusetts fue la primera en aceptar una mujer en $1884 .^{7}$ En Estados Unidos la situación local de cada estado, influyó notablemente el status de las mujeres dedicadas a la medicina. Al inicio del siglo XX las sociedades de médicas florecieron e impusieron sus propias reglas para la admisión, a la sombra de una especie de feminismo victoriano que pronto se fracturó. ${ }^{8}$ Después de la Primera Guerra Mundial la segregación declinó y aparentemente el gremio médico en general empezó a compartir los mismos espacios. Sin embargo, las asociaciones exclusivamente femeninas no dejaron de existir, así en 1919 se creó la Medical Women ’s International Association, en los años cuarenta, surgió la Pan American Medical Women's Alliance y en 1938, en Chile, nació la Agrupación Médica Femenina. ${ }^{9}$ Años antes, el 5 de mayo de 1926 en México se había formado la “Asociación de Médicas Mexicanas”, bajo la presidencia de la doctora Ursúa y dos vicepresidentas, Margarita Delgado y Ormesinda Ortiz Treviño. ${ }^{10}$

6 Martin Kaufman, “The admission of women to nineteenth-century american medical societies”, Bulletin of the History of Medicine 50, n. 2 (1976): 251-260.

$7 \quad$ Ibid., 260.

8 Ellen More, “The Blackwell Medical Society and the professionalization of women physicians”, Bulletin of the History of Medicine 61, n. 3 (1987): 603-628.

9 Teresa Ortiz Gómez, Ana Delgado Sánchez, Dolores Sánchez, Ana Távora Rivero, "Female professional identities and Spanish women doctors in late Francoism (1965-1978), en Mujeres en la ciencia y la tecnología: Hispanoamérica y Europa, coords. María Luisa Rodríguez-Sala y Judith Zubieta García (México, Universidad Nacional Autónoma de México, 2005), 121.

10 Expediente del doctor Everardo Landa. Archivo Histórico de la Academia Nacional de Medicina de México, ANMM. 


\section{Modelo de Estudio y Resultados}

Hasta el año 2006, la ANMM contaba con 536 académicos de los cuales 474 son hombres y 62 mujeres. En este grupo femenino se investigaron los siguientes parámetros: fecha de ingreso a la ANMM y edad en ese momento, edad actual, si tienen hijos y vida de pareja, el departamento y área donde se ubican y su pertenencia al Sistema Nacional de Investigadores (SNI). Muy pocas académicas no son médicas, tienen formación en biología, química, psicología o sociología y se ubican en los departamentos de Biología Médica o Salud Pública. Estas no se eliminaron de la muestra pues se consideró que no afectaban el objetivo de estudio.

Las mujeres integran tan solo el $11.5 \%$ de los académicos. De éstas, 16 son titulares (por antigüedad) y 46 son numerarias. Hasta finales del 2006, cuatro académicas habían fallecido: Rosario Barroso Moguel (1921-2006), Julieta Calderón de Laguna (1918-2001), Blanca Raquel Ordoñez de la Mora (1937-2002) y María de Lourdes Tamayo Sánchez (1930-2006); las que tampoco se sacaron de la muestra por no exigirlo los fines del estudio.

\section{Número de mujeres en la ANMM a partir del primer ingreso en 1957}

Desde 1957 cuando entró Rosario Barroso Moguel (el presidente era el doctor Bernardo Sepúlveda) hasta 1990, el ingreso fue más bien lento con amplios periodos en los que no hubo mujeres aceptadas (gráfica 1). En 1965 ingresó la segunda, Julieta Calderón de Laguna (presidente doctor Miguel Jiménez Sánchez) y María de la Soledad Córdova Caballero, la tercera en 1968 (presidente doctor Isaac Costero). Las dos primeras ya fallecieron y es difícil sustraerse a la mención de lo interesante y enriquecedor que fueron sus vidas. ${ }^{11}$ En 1979 se incorporaron cuatro doctoras (presidente doctor Jaime Woolrich Domínguez). Es claro que a partir de 1991 el ingreso se disparó, en 1994 se admitieron siete nuevas académicas (presidente doctor Carlos Campillo Serrano); cifra que se superó en 2001 cuando se incorporaron a la ANMM nueve mujeres (presidente doctor Julio Sotelo Morales).

\section{Gráfica 1. NÚMERO DE MUJERES QUE INGRESARON EN CADA DÉCADA (62 mujeres)}

11 Ana Cecilia Rodríguez de Romo, Gabriela Castañeda López, Rita Robles Valencia, Protagonistas de la Medicina Científica Mexicana, 1800-2006 (México, Universidad Nacional Autónoma de México, Facultad de Medicina, Plaza y Valdés, 2008). 


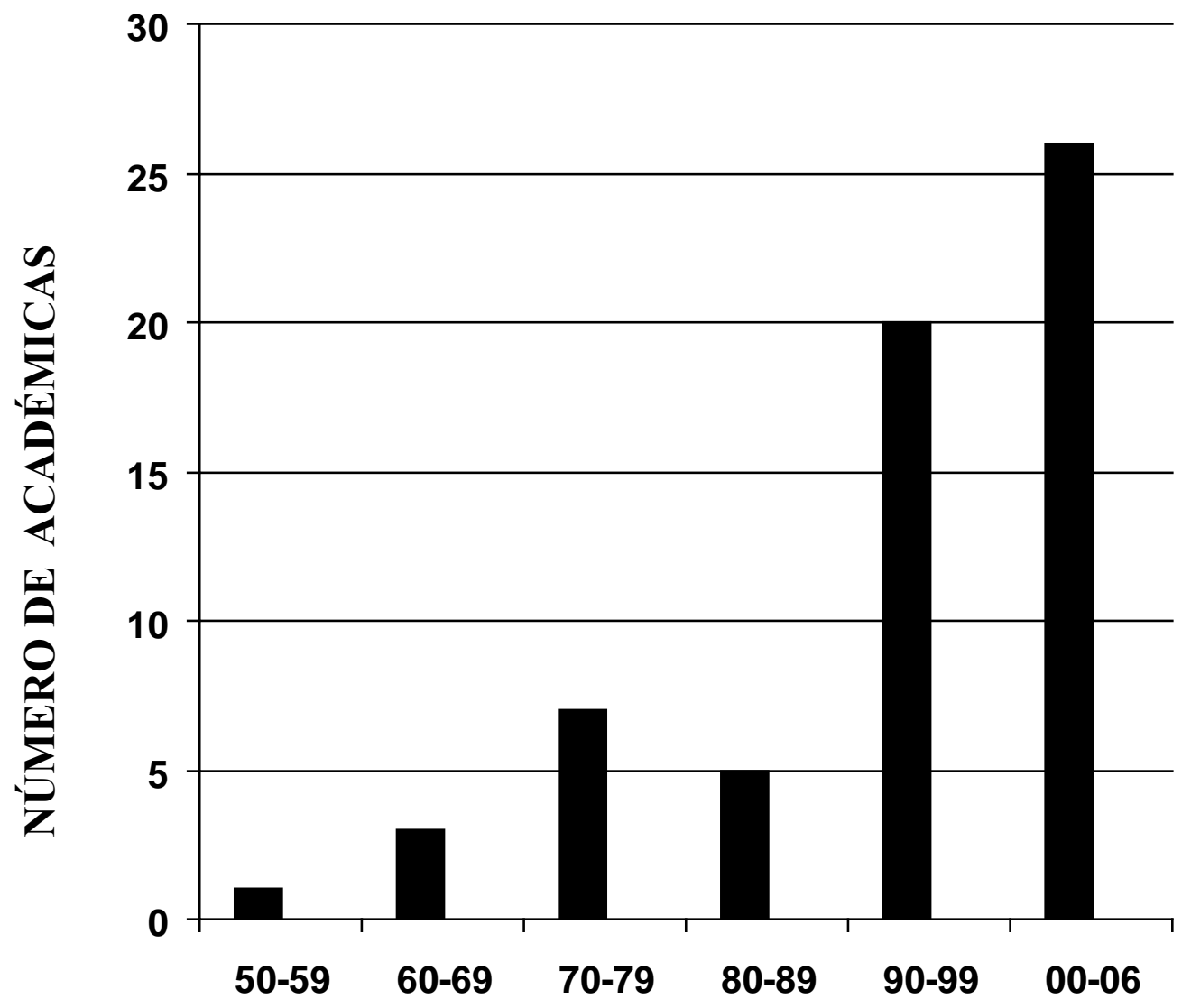

\section{AÑOS EN DÉCADAS}

\section{Edad de ingreso y edad actual}

De las académicas, 23 tenían entre 41 y 45 años de edad cuando ingresaron a la ANMM, 18 estaban entre 46 y 50 años (gráfica 2). Las únicas aceptadas en la década de sus treinta fueron Rosario Barroso que contaba con 34 años y Blanca Raquel Ordoñez de 32, también actualmente fallecida. Es interesante notar que éstas entraron a edad más temprana de la que ingresaron las que son más jóvenes ahora, cuando probablemente el acceso era más restringido. 
Indexaciones: Repositorio de Revistas UCR, DIALNET, Latindex, REDALYC Directorio y recolector de recursos digitales del Ministerio de Cultura de España, Directory of Open Access Journals. Diálogos Revista Electrónica de Historia ISSN 1409-469X. Número especial 2008. Dirección web: http://historia.fcs.ucr.ac.cr/dialogos.htm

Hasta finales del 2006, la edad de 42 de las 62 académicas fluctuaba entre 51 y 70 años. De éstas, 16 doctoras tenían entre 51 y 55 años y diez entre 66 y 70. La más joven era de 44 años y había una académica con 87 años.

De las cuatro académicas fallecidas, dos murieron a la edad de 82 años, una cuando tenía 76 y otra 65. 
919.CONGRESO

Indexaciones: Repositorio de Revistas UCR, DIALNET, Latindex, REDALYC Directorio y recolector de recursos digitales del Ministerio de Cultura de España, Directory of Open Access Journals.

Diálogos Revista Electrónica de Historia ISSN 1409-469X. Número especial 2008. Dirección web: http://historia.fcs.ucr.ac.cr/dialogos.htm

\section{Gráfica 2. EDAD DE INGRESO, ACTUAL Y DE FALLECIMIENTO}

\section{(62 mujeres)}

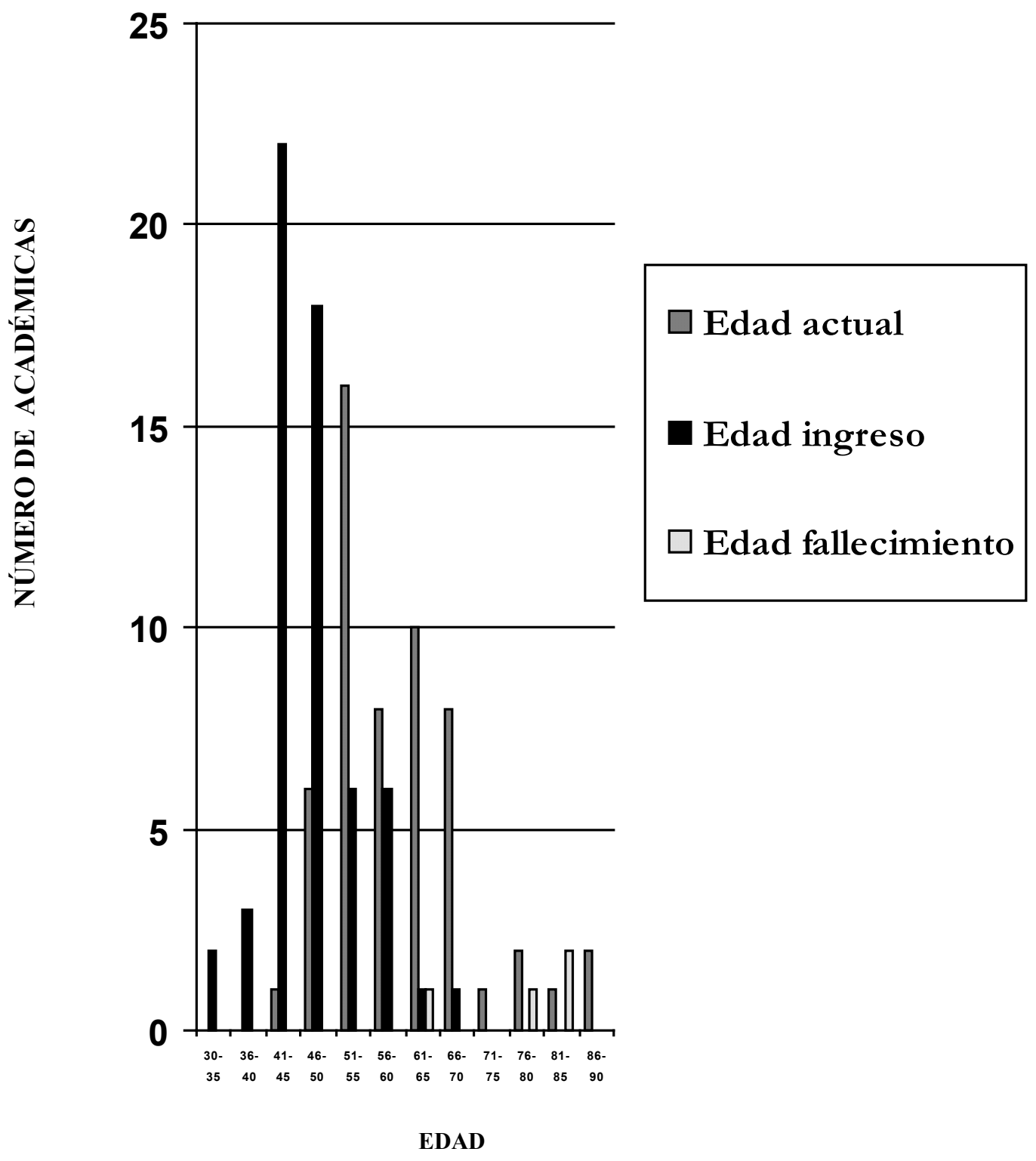




\section{El Sistema Nacional de Investigadores y la vida familiar}

El Sistema Nacional de Investigadores (SNI) fue creado en 1984 por Acuerdo Presidencial. Ante la grave crisis económica que entonces padecía el país, surgió como un instrumento del gobierno para estimular la investigación en México y apoyar a los científicos mediante estímulos económicos. El SNI tiene por objeto promover y fortalecer, a través de la evaluación, la calidad de la investigación científica y tecnológica, y la innovación que se produce en el país. ${ }^{12}$ La pertenencia al SNI no es obligatoria ni automática, está abierto a la comunidad de investigadores en activo de tiempo completo. ${ }^{13}$ La incorporación se da a través de un nombramiento en sus dos categorías: Investigador Nacional en uno de sus tres niveles y Candidato a Investigador Nacional. Dicho nombramiento se acompaña de un pago mensual, adicional al salario que percibe el investigador de su institución, fijado a la inflación, garantizando así su poder adquisitivo, no sujeto a gravámenes fiscales, otorgado de acuerdo al salario mínimo mensual y al nombramiento designado.

Dado que se busca premiar la productividad, tanto el nombramiento como el estímulo económico no son permanentes, se someten a una evaluación periódica por pares acorde al desempeño del investigador. ${ }^{14}$

En 1984, el investigador solicitaba su ingreso al SNI en una de sus tres áreas: Ciencias físicomatemáticas e ingeniería, Ciencias biológicas, biomédicas, agropecuarias y químicas y Ciencias sociales y humanidades; pero con el tiempo se han incorporado al proceso de evaluación nuevas áreas de conocimiento, en 2008 hay siete: Físico-matemáticas y ciencias de la tierra; Biología y química; Medicina y ciencias de la salud; Humanidades y ciencias de la conducta; Ciencias Sociales; Biotecnología y ciencias agropecuarias e Ingenierías.

La participación de la mujer en el SNI muestra un avance constante pero lento. La proporción ha aumentado al pasar del $20 \%$ en 1984 a $30 \%$ en $2003 .{ }^{15}$ Ser investigador nacional se ha convertido en una vía legítima que otorga un status a nivel individual y sobre todo, dentro

12 Diario Oficial, "Reglamento del Sistema Nacional de Investigadores (SNI)”, 21 de marzo de 2008.

13 Salvador Malo y Laura Rojo, "Estímulos para la productividad científica y las actividades docentes y artísticas en México”, Interciencia 21, n. 2 (1996): 71-72.

14 Salvador Malo, “El Sistema Nacional de Investigadores de México”, Interciencia 17, n. 6 (1992): 344-

347.

15 Una reflexión sobre el Sistema Nacional de Investigadores a 20 años de su creación (México, Foro Consultivo Científico y Tecnológico y la Academia Mexicana de Ciencias, 2005), 67 y 143. 
de la estructura institucional. ${ }^{16}$ Desde su creación el SNI ha modificado la forma de ingreso, actualmente es requisito indispensable poseer un grado académico y la pertenencia y nivel dependen principalmente del número y la calidad de las publicaciones y en menor grado de la formación de recursos humanos. En otras palabras, ingresar o mantenerse en el SNI, significa realizar investigación de calidad, lo cual precisa de mayor esfuerzo y puede provocar un gran estrés. Asumiendo esto, también se investigó cuantas académicas forman parte del SNI. Por otro lado, igualmente se obtuvo información respecto al número y edad de los hijos. Algunas fueron renuentes a informar su estado civil, por lo que este parámetro se eliminó de la muestra. Hubiera sido útil conocer el dato, considerando que también implica tiempo y esfuerzo compartir la vida cotidiana con una persona. Sólo 57 personas respondieron a las preguntas.

Suponiendo que cumplir con ambos parámetros: SNI e hijos es difícil ya que su confluencia incrementa el esfuerzo; la información indica que de 57 académicas, 29 (51\%) son investigadoras nacionales y tienen hijos, 12 (21\%) pertenecen al SNI pero no tienen hijos y solo 8 (14\%) no pertenecen al SNI y si tienen hijos; un número igual ni pertenece al Sistema ni tiene hijos.

Estos datos indicarían que ser académica y ser exitosa en la investigación científica, podrían ser dos situaciones que confluyen, pues el $72 \%$, o sea 41 académicas pertenecen al SNI y de éstas, 50 \% o la mitad también son madres. Llama la atención que de 57 mujeres, 20 (35\%) no tienen hijos.

\section{Area y Departamento en el que se ubican las académicas}

Para el análisis del departamento y área en el cual se ubican las académicas, se partió del supuesto que existen estilos de trabajo en cada subdisciplina. Por ejemplo, en la investigación básica, las investigadoras, que en su mayoría son independientes y titulares planean sus experimentos y hacen sus propios horarios. En cambio, en la clínica y la cirugía, existen los horarios hospitalarios de consulta y/o cirugía que no dependen del médico. Además, en muchos casos, la especialidad define la atención al paciente en la noche o en momentos inesperados, como sucede en la obstetricia o la pediatría. La consulta privada generalmente es en la tarde, cuando los niños ya no están en la escuela. En la sociomedicina usualmente hay horarios institucionales que la investigadora podría acomodar a su conveniencia.

En la ANMM hay cuatro departamentos: 1) Biología Médica, 2) Cirugía, 3) Medicina y 4) Salud 16 Teresa Corona, “La mujer en la ciencia”, Texto leído con motivo del In Memoriam a la doctora Rosario Barroso Moguel en el Instituto Nacional de Neurología y Neurocirugía, Ciudad de México, 2006. 
Pública y Sociología Médica. En el primero, generalmente se encuentran las investigadoras de las ciencias básicas, hasta 2006 contaba con 23 académicas de las cuales 20 están en el SNI. En Cirugía sólo existen cuatro académicas y ninguna de ellas está en el SNI. Al departamento de Medicina pertenecen 25 doctoras, 16 son investigadoras nacionales. Finalmente hay 10 mujeres en el Departamento de Salud Pública y Sociomedicina, cinco de ellas forman parte del SNI. En el cuadro 1 se incluyen todas las académicas según departamento y área en la ANMM.

\section{Cuadro 1. DEPARTAMENTO Y ÁREA EN LOS QUE SE UBICAN LAS ACADÉMICAS.}

\begin{tabular}{|c|c|c|c|}
\hline $\begin{array}{c}\text { BIOLOGÍA } \\
\text { MÉDICA }\end{array}$ & CIRUGÍA & MEDICINA & $\begin{array}{c}\text { SALUD PÚB. } \\
\text { SOC. MÉD. } \\
23\end{array}$ \\
\hline Laboratorio & Hospital & Hospital & Institución \\
\hline SNI 20 & SNI 0 & Consultorio & SNI 16 \\
\hline
\end{tabular}




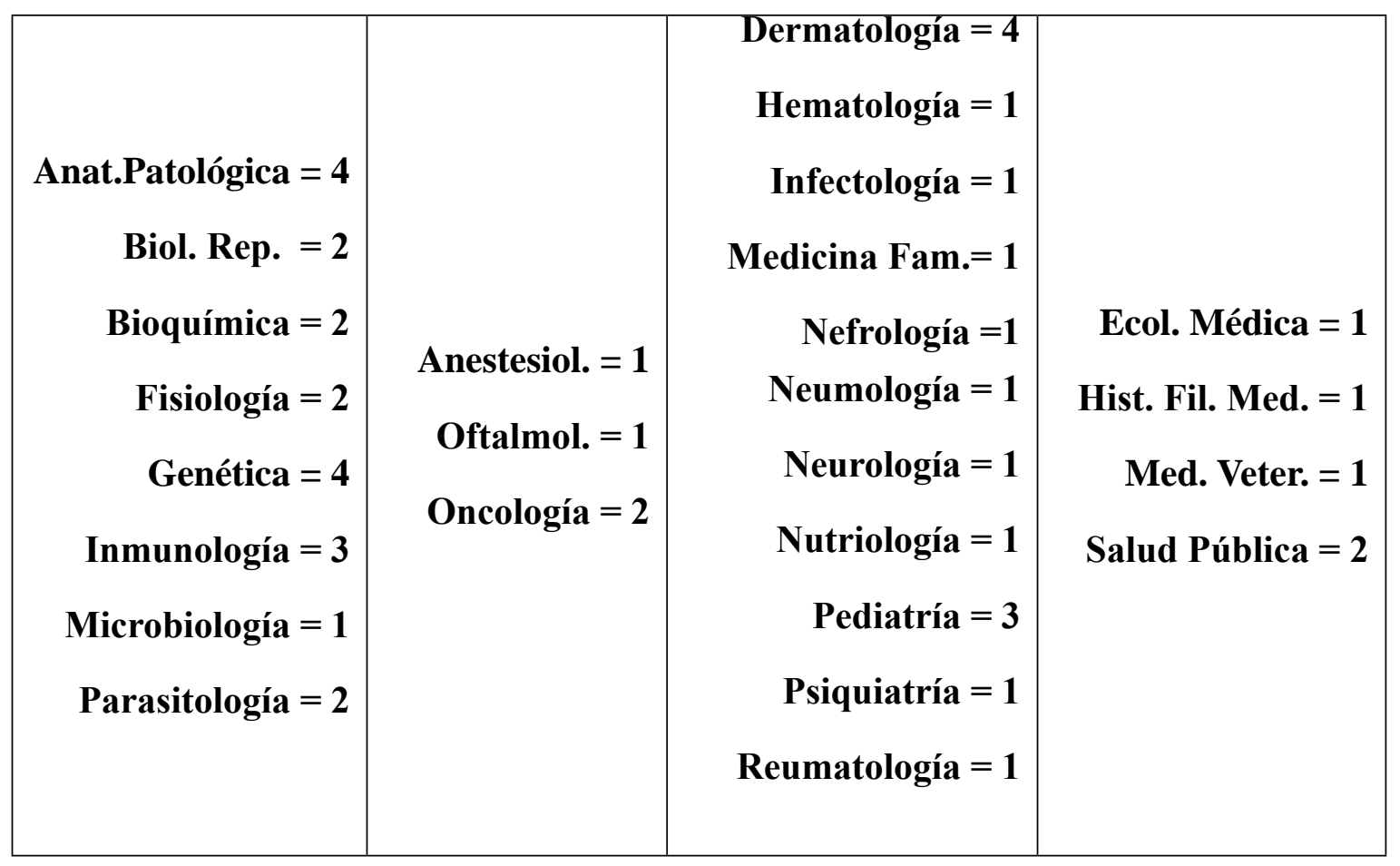

\section{Conclusiones}

Hasta el año 2006, las académicas en la ANMM representaban el 11.5\% de la población total. La entrada fue lenta entre 1957, año en que se admitió a la primera mujer, y 1990 (16 ingresos). A partir de entonces y hasta 2006, la admisión se aceleró incorporándose 46 mujeres. Lo anterior está de acuerdo con las tendencias mundiales actuales de aceptación de mujeres en clases de élite.

La edad promedio en el momento de integrarse a la ANMM es entre 41 y 45 años, es decir, 22 ingresos que representan el 35 \%. La cifra hace pensar que la mayoría entró una vez concluido el pico de la vida reproductiva. La edad de predominio actual es entre 51 y 55 años, hay 16 académicas en ese rango y representan el $27 \%$ de la población total. Veinticuatro doctoras, el $40 \%$, rebasan los 60 años, cuando los hijos ya son independientes. A este respecto cabría preguntarse a que edad la mujer alcanza su madurez académica. Más de la mitad, 29, combinan dos situaciones muy demandantes; tener hijos y satisfacer los requerimientos que su actividad profesional impone para pertenecer al SNI. 
Hasta 2006, las académicas en general eran mayores de 50 años y un buen número rebasan los 60, lo que habla más bien de una población madura y con experiencia.

Aunque un número importante no precisó su estado civil, por la forma de responder se puede suponer que predominan las casadas y las divorciadas.

1. Preocupación y responsabilidad familiar. Todas las académicas que tienen hijos y/o vida de pareja, dejan ver la importancia, pero sobre todo la preocupación que significa para ellas el buen desarrollo de su vida familiar, principalmente en el caso de tener hijos adolescentes. En este aspecto se puede concluir que es posible pero difícil para la mayoría de las académicas, compaginar la maternidad, la vida de pareja y la carrera académica. La mayoría piensa que la combinación lentifica su desarrollo profesional. ${ }^{17}$ En relación a los hijos, una académica divorciada dijo que tuvo que sacrificar la consulta privada de la tarde para estar más con sus hijas adolescentes pues si no, "la cosa iba por mal camino". Cuando a otra se le preguntó si era casada contestó: "hasta ahora si”.

2. Desempeño en áreas de horarios adaptables. La mayoría de las académicas están en el área de Biología Médica, son científicas independientes puesto que superaron la etapa de asistentes, colaboradoras o asociadas; ${ }^{18}$ esto les da libertad de trabajo. En número siguen las de la sección de Medicina que deben someterse a horarios hospitalarios y a veces "sacrifican" la consulta privada vespertina, sobre todo si tienen hijos adolescentes. Sólo hay cuatro académicas en Cirugía, pero en especialidades que les permiten escoger sus horas de ejercicio. Las del campo social pueden incluso trabajar en casa y construir su horario.

3. Área o disciplina en la ANMM. De acuerdo a los resultados, parecería que hay especialidades médico-científicas femeninas como dermatología y bioquímica y especialidades médicocientíficas masculinas como cirugía y neurofisiología. Es curioso mencionar que de las investigadoras solo dos están en fisiología, campo en el que pueden hacerse experimentos agudos largos. La proporción de investigadoras básicas en la ANMM es buena, 37\% y sería interesante

$17 \quad$ Estos resultados coinciden con los expresados en el estudio de Wendy Levinson, Susan W. Tolle, Charles Lewis, "Women in Academic Medicine: Combining career and family", The New England Journal of Medicine 321, n. 22 (1989):1511-1516.

$18 \quad$ No es lo mismo ser ayudante, asociada o titular en el laboratorio, situación bien demostrada en: Marianne Gosztonyi Ainley, "D’assistantes anonymes à chercheures scientifiques: une rétrospective sur la place des femmes en sciences”, en Des femmes dans les sciences. Cahiers de recherche sociologique (Québec, Département de Sociologie de l’Université du Québec à Montréal, 1986), 55-71. 
indagar las razones por las que escogieron el laboratorio para desarrollarse intelectualmente. No hay muchas científicas que sirvan de modelo en la historia, aunque es curioso observar la presencia de mujeres en el surgimiento de disciplinas científicas como la botánica o la geología, su predominio en la psicología, su influencia en la antropología y en la misma medicina. ${ }^{19}$ De las cirujanas, dos están en oncología, una en anestesia y otra en oftalmología. No hay ginecoobstetras a las que despiertan las pacientes en trabajo de parto. También hay que considerar el "estilo" de la subdisciplina. Las exigencias de la formación, tiempo o trato entre colegas, no son iguales para la neurocirugía, que para la cirugía dermatológica por ejemplo. La elección del tema de investigación, especialidad o campo de trabajo, no sólo depende de la experiencia personal, social y científica (es decir biográfica), parecería que también involucra cuestiones de género y educación. ${ }^{20}$

4. El poder. Sobre este aspecto, con los datos reunidos, es posible apuntar que fuera del ámbito de la ANMM, la mayoría de las académicas son investigadoras independientes (en todas las áreas), directoras o jefas de departamentos y servicios. Dos han sido directoras de Institutos Nacionales de Salud y recientemente una más fue nombrada también directora, sería interesante escuchar los problemas inherentes a su condición femenina que seguramente han confrontado como primeras mujeres líderes de grandes instituciones. Al seno de la propia ANMM ha habido secretarias generales y de actas así como tesoreras, pero nunca una presidenta, lo que no significa que no hayan mostrado interés por serlo. Dos mujeres han buscado la presidencia de la ANMM pero no fueron beneficiadas con los votos necesarios. Estudiando las sociedades españolas de salud pública, Teresa Ortiz ha encontrado que los puestos donde se concentran las mujeres son las tesorerías y las vocalías y que no hay presidentas. ${ }^{21}$ Sin embargo, el Colegio de Médicos de Madrid tuvo la primera presidenta en el año 2000, la doctora Juliana Fariña González, jefa del servicio de anatomía patológica del hospital Clínico.

Los estudiosos de género han propuesto el concepto "techo de cristal”, ${ }^{22}$ que es una especie de superficie superior invisible que las mujeres se autoimponen en su carrera laboral. Se refiere 19 Eulalia Pérez Sedeño, “Una ciencia ¿de quién y para quién?”, Ciencias 77 (2005):22.

20 Teresa Ortiz Gómez, "El papel del género en la construcción histórica del conocimiento científico sobre la mujer", en La salud de las mujeres: hacia la igualdad de género en salud, ed. Elvira Ramos (Madrid, Ministerio de Trabajo y Asuntos Sociales-Instituto de la Mujer, 2002), 31.

21 Teresa Ortiz Gómez, Johanna Birriel Salcedo, Rosa Ortega del Olmo, “Género, profesiones sanitarias y salud pública”, Gaceta Sanitaria 18, supl. 1 (2004):192.

22 Mabel Burin, “Género y Psicoanálisis: Subjetividades femeninas vulnerables”, en Género, psicoanálisis y subjetividad, comps. Mabel Burin y Emilie Dio Bleichmar (Buenos Aires, Paidos, 1996), 79. 
a la decisión personal de rechazar las oportunidades que se presentan para ocupar puestos de responsabilidad y decisión, por anteponer la vida familiar y no verse confrontada a situaciones que involucren aspectos emocionales. También apuntan que: "las mujeres temen ocupar posiciones de poder" o "no les interesa ocupar puestos de responsabilidad". ${ }^{23}$ Los resultados de este estudio, muestran que estos principios no se aplican a todas las académicas. La mayoría planifican su carrera, muestran ambición y se capacitan para lograr sus fines.

\section{Discusión}

Hasta el año de 2006, las mujeres constituían el 11.5\% de la población total de la ANMM. El trabajo ofrece explicaciones a esta baja proporción, pero una muy clara y que no se puede soslayar, es el pobre ingreso de mujeres a las escuelas de medicina, cuando menos hasta mediados del siglo veinte. También es un hecho que a las mujeres se les exige más cuando incursionan en ámbitos inicialmente masculinos como la medicina, que todavía no hay suficientes modelos femeninos con los que identificarse, que entre las mismas mujeres hay rivalidad y que las doctoras pueden ser muy calificadas, pero de acuerdo a la bibliografía en general, prefieren ubicarse en campos si no menos atractivos, si quizá menos desafiantes.

En las élites de mujeres, principalmente se encontraron estudios con ejecutivas e ingenieras y los resultados son muy semejantes a los pocos que hay en la medicina. Casi todos los directores generales con capacidad de decisión económica en las grandes compañías son hombres, la relación en los niveles más altos es 1 a 14 (regresando a las médicas, la ANMM no ha tenido presidentas, pero si ha habido directoras de escuelas de medicina). En la economía existe lo que llaman segregación vertical, hay menos mujeres en los más altos niveles (en la medicina otra vez, son muy pocas las directoras de escuelas e institutos) y segregación horizontal, las mujeres se ubican en las ramas consideradas de menor relevancia como recursos humanos. ${ }^{24}$ Las excepciones no dejan de ser atractivas, por ejemplo, Carly Fiorina, fue la protagonista de una revolución innovadora que convirtió a Hewlett Packard (HP), de fabricante de impresoras a proveedor de servicios globales de tecnología. ${ }^{25}$ Sus detractores atribuyen a la ambición y no a su capacidad los beneficios que aportó a HP y el escándalo de su caída en 2005, quizá no habría sido tanto, si se hubiera tratado de un hombre.

$23 \quad$ Ibid., 82 .

24 Zabludovsky, 209.

25 Sacnicte Bastida y Juan Antonio Oseguera, “Poder, amargo poder”, Revista Expansión (marzo 2005): 70. 
La participación como dirigentes en puestos de representación política y gremial es baja. Para 1996, solo había tres mujeres en las direcciones generales de las cámaras de la CONCAMIN y estaban en embellecimiento físico, industria cinematográfica y la industria de las lavanderías. ${ }^{26}$ Igual que las empresarias, las médicas de la ANMM debieron adquirir experiencia y un determinado nivel antes de pretender integrarse a la corporación. También tener o haber tenido una situación económica relativamente privilegiada que le haya permitido estudiar y después sacrificar horas de consulta o escoger horarios sin merma o dependencia total del ingreso económico. Un alto índice de las empresarias no tiene compañero (solteras o divorciadas). Aunque no se exploró, por algunas respuestas se intuyó que la diferencia de ingreso económico a favor de la mujer o el éxito profesional reflejado en la aceptación por la ANMM, tuvo o tiene alguna influencia negativa en la vida de pareja de algunas académicas (situación semejante sucede con las empresarias). Como ya se mencionó, no son abundantes los estudios con médicas. Pero vale la pena mencionar el realizado en 1999 con la población médica en Noruega ya que incluyó a todos los doctores que en ese momento tenía el país y las preguntas que se plantearon. ${ }^{27}$ Entonces los noruegos contaban con 13,844 médicos de los cuales 3,939 eran mujeres y 10,131 hombres. El objetivo del trabajo fue explorar la relación entre hombres y mujeres en las posiciones de liderazgo. Además de las limitaciones individuales como las familiares y no ambicionar ser líder, se puso atención en las limitaciones estructurales para explicar por qué las médicas que aspiran a posiciones altas encuentran barreras invisibles. Encontraron que los consejos de patrones y amigos contribuyen al estructurar el "techo de cristal", concepto explicado en líneas anteriores. Aunque en Noruega la proporción de mujeres en la medicina se incrementa, éstas tienden a establecerse en trabajos rutinarios y mal remunerados, además los estereotipos se mantienen, por ejemplo, no tenían neurocirujanas. Sin soslayar la discriminación, la baja proporción de mujeres en las posiciones de autoridad, para el estudio reflejó un efecto sistemático donde la mujer simplemente no busca las posiciones de liderazgo. También mostró que es difícil apartarse de la tradicional actitud jerárquica que caracteriza a la medicina, asociada a la edad y al género, y que las mujeres enfrentan un dilema cuando se trata de escoger entre los puestos asociados al poder e influencia, y la familia (matrimonio y niños) y responsabilidades emocionales. De igual modo, en sus decisiones consideran las largas horas de trabajo y las guardias, así como la carrera de la pareja.

$26 \quad$ Zabludovsky, 212.

27 Kari J. Kvaerner, Olaf G. Aasland, Grete S. Botten, "Female medical leadership: cross sectional study", British Medical Journal 318 (1999): 93-94. 
Volviendo al caso mexicano, la investigación contestó las preguntas que en un principio se plantearon pero también abrió otras interrogantes, por ejemplo:

1. ¿La muestra puede considerarse representativa de la población femenina en las sociedades académicas de las llamadas ciencias duras? o, ¿sólo es reflejo de la medicina en particular?

2. ¿Cuáles son las motivaciones de la muestra estudiada para entrar a la ANMM? Posibilidades podrían ser: la vinculación con la ANMM, conciencia colectiva, compromiso, formar parte de redes, contactos o gremios, la ambición intelectual o el deseo de alcanzar un status privilegiado y lo que esto implica.

3. ¿Las académicas de la ANMM tienen algo que las haga diferentes a otras profesionales? Y si es así; ¿ ¿en dónde radican esas diferencias? Aunque aquí aplicaría bien eso de que no son todas las que están y no están todas las que son, ¿̇uvieron la oportunidad de una mejor preparación?, ¿su educación o personalidad las hace buscar ser diferentes, el prestigio y/o el poder? o ¿desean la superación personal, la satisfacción intelectual o persiguen ideales?

4. ¿cómo explicar que en este momento la población femenina es mayor que la masculina en las escuelas de medicina?

Todas son preguntas dignas de respuesta, que dan la pauta para un siguiente estudio.

\section{Notas biográficas de la primera médica mexicana y las dos primeras académicas,} las tres fallecidas

Matilde Petra Montoya y Lafragua ${ }^{28}$ (n. Ciudad de México, 14 de marzo de 1859; m. Ciudad de México, 26 de enero de 1938) Ginecología, Obstetricia

Su nombre completo era Matilde Tiburcia Valeriana. Ingresó a la Escuela Nacional de Medicina en 1870. En 1871, afectada por un padecimiento no especificado, abandonó sus estudios y viajó a la ciudad de Cuernavaca, a cien km. de la capital del país, donde radicó por un tiempo. El entonces jefe político de aquel distrito la invitó a ejercer la obstetricia pero ella se rehusó por no contar con el título de autorización; con este motivo se formó un jurado que la examinó y

28 Archivo Histórico de la Facultad de Medicina de la UNAM. Fondo Escuela de Medicina y Alumnos, leg. 56, exp. 47, f. 12; Diccionario Porrúa de historia, biografía y geografía de México, $6^{a}$ ed. (México, Porrúa, 1995), T. III, 2344-2345; Aurora Tovar Ramírez, Mil quinientas mujeres en nuestra conciencia colectiva: catálogo biográfico de mujeres de México (México, Documentación y Estudios de Mujeres (DEMAC), 1996), 433-434; Ana María Carrillo, Matilde Montoya: primera médica mexicana (México, Documentación y Estudios de Mujeres (DEMAC), 2002); Leticia Rodríguez Pimentel, Rodolfo Silva Romo, “Aportaciones de la mujer a la medicina”, Gaceta Médica de México 139, n. 6 (2003): 635-638. 
certificó que contaba con los conocimientos necesarios para dedicarse a la obstetricia.

Matilde Montoya permaneció en Cuernavaca ejerciendo su profesión con la autorización del jefe político hasta mayo de 1872, fecha en que regresó a la ciudad de México y reingresó a la Escuela Nacional de Medicina, donde siguió estudiando. En 1873 se recibió como partera y realizó prácticas en el Hospital de San Andrés. Al mismo tiempo aprendió cirugía menor y en su casa estudió latín, griego y matemáticas, dirigida por dos profesores particulares.

Debido a una enfermedad "desconocida", en 1875 se trasladó de la capital a la ciudad de Puebla donde, una vez restablecida su salud, comenzó a ejercer su profesión dedicándose exclusivamente a operaciones de obstetricia y enfermedades de señoras. Adquirió gran prestigio, pero la oposición de médicos que la calumniaron y difamaron, la obligaron a abandonar Puebla y radicarse en la ciudad de Veracruz. Regresó a Puebla en 1880. En 1881 comenzó a asistir a la Escuela Nacional de Medicina y presentó su examen profesional el 24 y 25 de agosto de 1887 con la tesis Técnicas de laboratorio en algunas investigaciones clínicas, convirtiéndose en la primera médica mexicana graduada oficialmente. Se dice que en la defensa de su examen estuvo presente el Presidente Porfirio Díaz.

Al concluir los estudios profesionales se dedicó a la consulta particular para señoras y niños en la ciudad de México.

Tuvo la satisfacción de celebrar su jubileo profesional en agosto de 1937, recibiendo el homenaje de la Sociedad de Médicas Mexicanas, de la Asociación de Universitarias Mexicanas y del Ateneo de Mujeres, a cuya celebración se asociaron otras agrupaciones científicas de México.

Rosario Barroso Moguel $^{29}$ (n. Estado de Oaxaca, 5 de octubre de 1921; m. Ciudad de México, 21 de enero de 2006) Patología

Obtuvo el título de médica cirujana el 7 de junio de 1945 por la Universidad Nacional Autónoma de México (UNAM), con mención honorífica. Siendo estudiante trabajó en el Laboratorio de Estudios Médicos y Biológicos de la misma UNAM. Realizó la maestría en Ciencias Médicas, se especializó en anatomía patológica en 1962 y fue una de las primeras en cursar un doctorado en la Facultad de Medicina (UNAM) que obtuvo en 1968. Fue miembro fundador y titular del Consejo Mexicano de Médicos Anatomopatólogos en 1963.

29 Tovar Ramírez, 64-65; “In memoriam. Dra. Rosario Barroso Moguel”, Gaceta Salud n. 44 (2006):12; Julio Sotelo, “In memoriam. Doctora Rosario Barroso Moguel”, Gaceta Médica de México 142, n. 3 (2006): 271272. 
Se entrenó con los mejores patólogos de su tiempo. De 1948 a 1950 realizó una estancia en el Pathology Department del Columbia Medical Center (Nueva York) con el doctor P. Scout; en el Departamento de Patología del Veterans Administration Hospital (Nueva York) con el doctor Gordon; en el Departamento de Patología del Memorial Hospital de Nueva York, con los doctores Spitz y Allen, y finalmente en el Armed Forces Institute of Pathology (Bethesda, Maryland, EU) con el doctor Ash.

En 1945 fundó el Departamento de Anatomía Patológica en el Hospital de Enfermedades de la Nutrición. Fue técnica en el Departamento de Anatomía Patológica del Instituto Nacional de Cardiología, investigadora de tiempo completo en el mismo Departamento (1952-1973) y luego subjefa hasta los últimos meses de 1973.

En la UNAM desempeñó diversos cargos: profesora por oposición de anatomía patológica en 1962, profesora adjunta de anatomía patológica en la División de Estudios Superiores (19481972), profesora titular de histología (1965-1970), miembro del Consejo Técnico de la Facultad de Medicina (1958-1963), profesora titular en el curso de patólogos (1960-1965) y profesora de histopatología general en el curso de profesores en anatomía patológica (1965-1968).

Fue además profesora titular de histología normal y de anatomía patológica en el Instituto Politécnico Nacional (1965-1970) y estuvo a cargó de las cátedras de histología y fisiología en la Escuela de Enfermería del Instituto Nacional de Cardiología.

En el Instituto Nacional de Neurología y Neurocirugía, junto con el doctor Isaac Costero, fundó en 1974 el Laboratorio de Neurobiología Celular (1973-1979), nombre que posteriormente cambió a Laboratorio de Neuromorfología Celular; ocupó la jefatura del mismo de 1983 a 2005. Ahí llegó a ser investigador titular “C” de la Secretaría de Salud (1984-2005), así como investigadora nacional Nivel III del SNI (1997-2005).

Elaboró 28 películas sobre cultivo de tejidos conjuntivo, miocárdico y nervioso en colaboración con los doctores Isaac Costero, Charles M. Pomerat y Agustín Chávez. Dirigió numerosas tesis de grado y posgrado. Son clásicos sus reportes de neurohistología del cuerpo carotídeo y los estudios sobre alteraciones del sistema nervioso secundarios a la administración de cocaína.

En un tiempo en que la medicina era una profesión mayoritariamente masculina, se distinguió entre sus colegas patólogos varones, infundiendo en ellos respeto y admiración. Nombrada “La mujer del año 1988” por el Patronato Nacional de la Mujer del Año, A.C. Fungió como vicepresidenta y presidenta de la Rama Mexicana de la International Academy of Pathology de 
1964 a 1967. Perteneció a distinguidas sociedades médicas de su especialidad y publicó más de 200 trabajos científicos. Autora de dos libros: Biología de los gliomas y en coautoría con Víctor Romero Díaz Thinner: inhalación y consecuencias (1988). Era políglota y una espléndida dibujante.

Julieta Calderón Gené ${ }^{30}$ (n. San José, Costa Rica, 1918; m. Ciudad de México, 3 de febrero de 2001) Cancerología, Citología Exfoliativa

Estudió en la Facultad de Medicina de la Universidad Nacional Autónoma de México (UNAM). Es considerada pionera de la citología exfoliativa en México.

Durante la década de los cuarenta fue médica asistente del Servicio de Cáncer Ginecológico en el Hospital General de la ciudad de México y el Centro de Detección y Diagnóstico del Servicio de Cáncer Mamario y Ginecológico de la Secretaría de Salubridad y Asistencia (SSA). Después de su adiestramiento (1947-1948) en el Memorial Hospital de Nueva York, con los doctores Papanicolaou y Cross, y la bióloga Ruth Graham, inició el ejercicio de su especialidad. Fundó y fue jefa del Laboratorio de Citología Exfoliativa de los hospitales "Dr. Jesús Alemán Pérez" (1950), Instituto Nacional de Cancerología y Hospital "20 de Noviembre”. Participó en la organización de los servicios médicos del Instituto de Seguridad Social y Servicios para los Trabajadores del Estado (ISSSTE) y se desempeñó como médica adjunta del Departamento de Radioterapia del Instituto Nacional de Cancerología.

Realizó estudios de posgrado en el Laboratorio de Anatomía Patológica del Instituto Nacional de Radioterapia y el Massachussets General Hospital; fue research fellow del Vicent Laboratory.

En el Hospital Juárez impartió cursos de cancerología ginecológica y dirigió la campaña contra el cáncer. Enseñó oncología en la Escuela Superior de Medicina Rural del Instituto Politécnico Nacional. En la UNAM perteneció a la División del Doctorado de la Facultad de Medicina, impartió radiología, coordinó cursos de ginecología y obstetricia, y fue profesora titular de las mismas disciplinas. Jefa del curso de posgrado de médicos citólogos del ISSSTE (1969-1990) y del curso de citología del Hospital “20 de Noviembre”.

Recibió la adscripción al Programa de Becas de la OEA para adiestramiento de médicos

30 "Pionera de la citología exfoliativa en México. En memoria de la doctora Julieta Calderón Gené de Laguna”, Gaceta de la Facultad de Medicina UNAM (25 de febrero 2001). 
citólogos; destacó su interés en la formación de alumnos dentro del ámbito médico nacional y latinoamericano.

En la década de los sesenta fue miembro del Consejo Consultivo del Hospital de la Mujer y del Instituto Nacional de Cancerología. Como en esos años la tecnología era ya una herramienta indispensable para la medicina, la doctora Laguna se trasladó a Chicago para estudiar un diplomado sobre automatización de la citología del cáncer uterino.

En 1976 fue nombrada jefa de la Oficina Normativa de Oncología del ISSSTE. También ocupó la Dirección General de Lucha Contra el Cáncer de la SSA. En 1978 aceptó un puesto directivo en el Programa de Detección Oportuna del Cáncer Cérvicouterino en la misma SSA. En 1983 presidió la Comisión de Oncología del ISSSTE.

Autora de múltiples trabajos en libros y revistas relacionadas con la citología hormonal, morfología, citología, el cáncer cérvicouterino y los cambios provocados por su tratamiento con radiación.

Perteneció a la Academia Internacional de Citología Ginecológica (actualmente Academia Internacional de Citología), la Sociedad Latinoamericana de Citología, la Asociación Mexicana de Citología Exfoliativa, además de otras agrupaciones científicas nacionales e internacionales, algunas de las cuales presidió.

Su fructífera vida profesional la llevó a recibir numerosas distinciones como el nombramiento de Mujer del Año.

\section{Agradecimientos}

Agradecemos a las Académicas que amablemente contestaron nuestras preguntas, permitiendo así la realización de este trabajo. Igualmente gracias a las señoras Elizabeth Gutiérrez Ochoa y Lydia Cornejo Ochoa por el material facilitado, Cecilia Rivera por el apoyo técnico, los doctores Teresa Corona, Ana Flisser, Héctor Pérez-Rincón, Ranulfo Romo y Julio Sotelo por sus comentarios. 